\title{
No-Reference Image Quality Assessment Based on Visual Perception
}

\author{
Ni Jun ${ }^{a}$, Li ZiYin ${ }^{\text {b }}$, Chen HuaCai ${ }^{\mathrm{C}}$ \\ College of Optical and Electronic Technology, China JiLiang University, Hangzhou, China \\ anijun@cjlu.edu.cn, bliziyin@cjlu.edu.cn, 'chuacaichen@cjlu.edu.cn
}

\begin{abstract}
Keywords: Image quality assessment; no-reference image; visual perception; automatic focusing;
\end{abstract} Image Sharpness Metric

\begin{abstract}
No-reference image quality assessment is an important issue for video compression and communication. This work presents a no-reference objective image/video sharpness method based on visual perception metric (VPM). The algorithm gets image typical edge and edge width firstly, and then gets gray contrast of typical edge region, finally utilizes these factors to integrate a probability summation assessment model. The proposed metric is able to predict the amount of sharpness in image with different content. Experimental results show that this method is consistent with subjective assessment of human being and can be use to describe the visual perception of image effectively.
\end{abstract}

\section{Introduction}

Image quality assessment plays an important role in various networks or communication applications, such as video image acquisition and transmission, the choice of parameters in coding systems, and the performance comparison of different image compression algorithms [1]. Generally, image quality assessment methods can be classified as subjective methods and objective methods[2]. According to the original reference image information used in algorithm, there are three types of methods that are used for objective image quality evaluation: full-reference (FR), reduced-reference (RR) and no-reference (NR) [3]. To networks or communication applications, most images or video are only being captured when received, hence inaccessibility of the original reference image information[4], It is difficult to assess the image quality[5], Therefore, there is an unquestionable need to develop metrics that closely correlate with human perception without needing the reference image[6][7]. We develop a sharpness metric based on the shape of local kurtosis at the edge regions and present a new approach based on visual perception, which does not require a reference image and uses a new sharpness scale for images edge. The new method is the spatial domain approaches that overcomes the disadvantages of frequency domain approach, and also eliminates the high sensitivity to asymmetric kurtosis distributions. Extensive experiments on a blur image database justify the proposed algorithm an effective NR image quality assessment method. This paper is organized as follows. Section 2 introduces the existing method to assess image quality. Section 3 presents image sharpness metric based on Visual Perception. Section 4 includes results using the improved metric, and Section 5 presents conclusions and ideas for future research.

\section{Existing algorithms for image quality assessment}

Image quality metrics be used currently are subjective method and objective method. Subjective method is that observer give the sharpness assessment score for the image by their experience. Subjective method has two yardsticks: absolute assessment and relative assessment. Subjective method is difficult to definition by mathematic model. So it is conditionality used in practical application. Objective method is that use mathematic measure gets score about image sharpness. The most widely used objective image quality metrics, namely mean square error (MSE) and peak signal to noise ratio (PSNR) [6]

MSE and PSNR are easy for calculation but they are widely criticized because they are usually not correlation well with perceived sharpness measurement. A great deal of research has been made to develop effective objective image sharpness metrics that incorporate perceptual sharpness measures during the past time. But results were depressed. Some sharpness metrics pay more attention on 
human visual system (HVS) characteristics. But most of the proposed image sharpness assessment approaches require the original image as a reference. Nevertheless, human eyes make judgments for image quality and do not need reference image. Human observers can easily assess the quality of distorted images without using any reference image. By contrast, designing objective NR quality measurement algorithms is a very difficult task. This is mainly due to the limited understanding of the HVS, and it is believed that effective NR quality assessment is feasible only when prior knowledge about the image distortion types is available. Although only a limited number of methods have been proposed in the literatures for objective NR quality assessment, this topic has attracted a great deal of attention recently.

\section{Image quality assessments Based on Visual Perception}

By identifying gradient characteristics in image, human can assess the sharpness degree of distorted images without using any reference image easily and quickly. The distance that gradient along the normal of edge present the sharpness degree. This section, a simply algorithm is proposed which used to calculate the shape of edge transition. By the parameter of edge kurtosis value, the degree of image sharpness can be assessed without need any reference images.

\subsection{Direction and position of edge}

For human being, the sensitivity of sharpness is come from typical edge in image. To measure gradient shape parameter, the direction and position of edge must be detected first. Gradient in image is got by operators:

$$
D(x, y)=\frac{1}{12} \begin{array}{|c|c|c|}
\hline-1 & -2 & -1 \\
\hline-2 & 12 & -2 \\
\hline-1 & -2 & -1 \\
\hline
\end{array} * f(x, y)
$$

To reduce the complexities of calculation, four directions detection operator used in this algorithm.

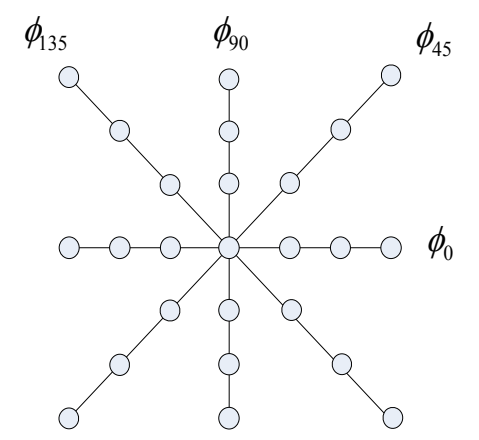

Fig. 1 four direction operators

Four direction operators slide on the gradient matrix of image. By calculating the sum value of the seven gradients along the four directions, it selects the biggest value as the position of typical edge and subscript of $\phi$ represents the direction of edge.

\subsection{The parameter of edge transition zone}

The gradient quantities' attribute along the normal of edge represent the sharpness degree. Here, the algorithm set three parameters for represent the gradient distributing along the edge transition zone.

At the typical edge position $P(x, y)$, the algorithm selects seven pixels $p_{i}(i=0,1 \ldots 6$,$) along the$ normal line of edge and calculates the relative gradient $t_{i}(i=0,1 \ldots 5$,

Relative gradient $t_{i}$ :

$$
t_{i}=\operatorname{abs}\left(p_{i}-p_{i+1}\right)
$$


Then it get the different value between the biggest and the smallest of $t_{i}(i=0,1 \ldots 5$, .

$$
S=t_{\max }-t_{\min }
$$

Next it judge whether the effective edge exist or not. If $S_{\max }>T$, then, effective edge exist. Here, $T$ is threshold value and $T=1.5$ average $\left(t_{i}\right)$.

Select the biggest value $t_{\max 1}: \quad t_{\max 1}=\max \left(t_{0}, t_{1}, t_{2} \ldots t_{5}\right)$

And select other two biggest value $t_{\max 2}$ and $t_{\max 3}$ among $t_{i}$ :

$$
\begin{aligned}
& t_{\max 2}=\max \left[\left(t_{0}+t_{1}\right),\left(t_{1}+t_{2}\right),\left(t_{2}+t_{3}\right),\left(t_{3}+t_{4}\right),\left(t_{4}+t_{5}\right)\right] \\
& t_{\max 3}=\max \left[\left(t_{0}+t_{1}+t_{2}\right),\left(t_{1}+t_{2}+t_{3}\right),\left(t_{2}+t_{3}+t_{4}\right),\left(t_{3}+t_{4}+t_{5}\right)\right]
\end{aligned}
$$

Set three parameters $p_{1}, p_{2}$ and $p_{3}$ represent the gradient distributing.

$$
p_{1}=t_{\max 1} / \chi \quad ; p_{2}=t_{\max 2} / \chi ; p_{3}=t_{\max 3} / \chi
$$

Here $\chi$ is sum of $t_{0}$ to $t_{5}, \quad \chi=\operatorname{SUM}\left(t_{0} \ldots t_{5}\right)$

The proposed method uses above three parameters to express the transition character of typical edge in current image. The degree of image sharpness classification can be described by Eq.8.

$$
F=\alpha p_{1}+\beta\left(p_{2}-p_{1}\right)+\delta\left(p_{3}-p_{2}\right)
$$

$\alpha, \beta, \delta$ is weight value for parameters $p_{1}, p_{2}$ and $p_{3}$.

Under the condition of image have appropriate contrast, the image will look as in focus when the value of parameter $F$ arrive a threshold value.

\section{Experimental Results}

In order to verify proposed image quality assessment algorithms effective, we select the Texas' blur image database[8]. Two serial of images which from in focus to out of focus is show in fig2 and fig3. Fro human eyes, degree of sharpness in image is decreasing from fig2 (a) to fig2 (e). Also, from fig3 (a) to fig3 (e)

In experiment, we set $\alpha=0.5, \beta=0.35, \delta=0.15$. White frame in image is ROI (Region of Interesting). In ROF, we calculate the quality assessment parameter $F$ by Eq. 8

One serial of images (monarch) show in Fig.2

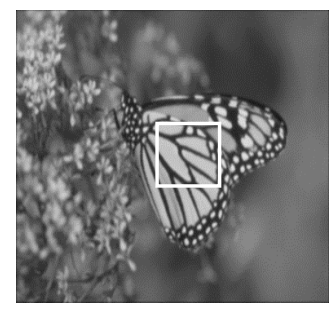

(a) $\begin{aligned} F & =0.5784 \\ \sigma & =0.906218\end{aligned}$

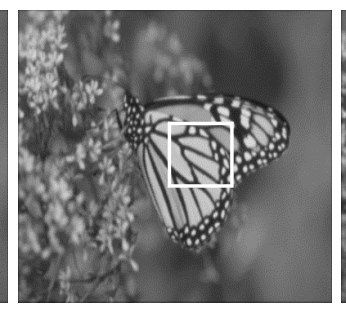

(b) $F=0.5052$ $\sigma=1.708303$

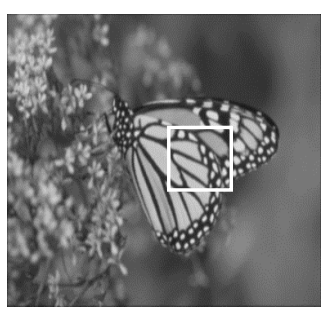

(c) $F=0.4618$ $\sigma=1.851533$

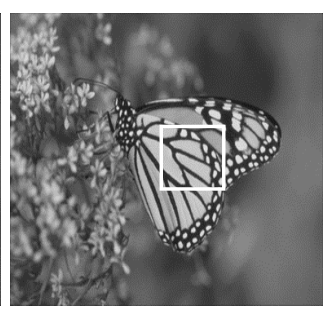

(d) $F=0.3724$ $\sigma=2.854139$

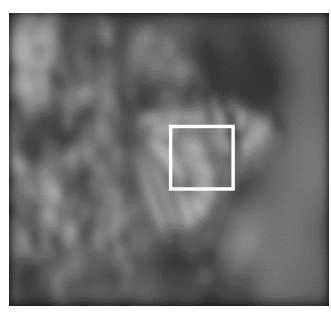

(e) $F=0.1355$ $\sigma=11.333325$

Fig.2 Serial of images with different degree of sharpness (monarch) 
Another serial of images (plane) show in Fig.3

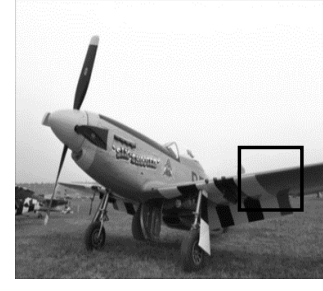

(a) $\begin{aligned} F & =0.5876 \\ \sigma & =0.562467\end{aligned}$

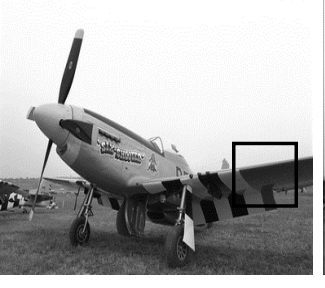

(b) $\begin{aligned} F & =0.5412 \\ \sigma & =1.078093\end{aligned}$

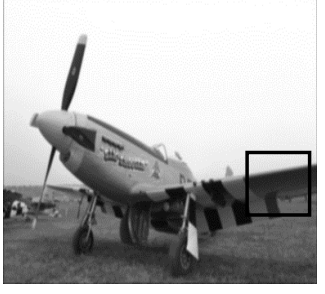

(c) $F=0.5038$ $\sigma=1.708303$

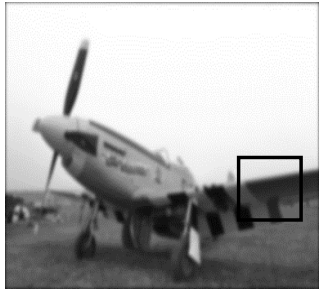

(d) $F=0.3701$ $\sigma=3.312473$

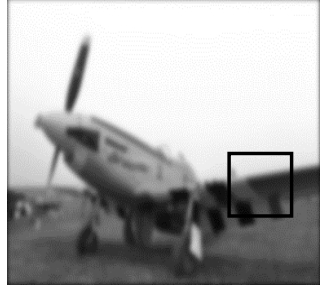

(e) $F=0.2619$ $\sigma=4.916644$

Fig.3 Serial of images with different degree of sharpness (plane)

Texas' gaussian-blurred image database were filtered using a circular-symmetric 2-D gaussian kernel of standard deviation sigma. The value of sigma $(\sigma)$ is given in Fig.2 and Fig.3 Exponent $F$ can represent the image sharpness degree. Variation value of $F$ is between zero to one. As for in focus image, the ideality value of $F$ is one.

As showed in fig. 2 and fig. 3, exponent $F$ is change according to Gaussian parameter $\sigma$. These parameters indicate that exponent $F$ can Assess image sharpness degree without need reference images.

To measure how well the metric value correlate with the provided MOS value, we follow the Video Quality Expert Group (VQEG) report [9] where several evaluation metrics are proposed. Here, we use PSRN and SSIM for test. Note that a logistic fitting function is used, as suggested in [9], to provide a nonlinear mapping between the objective/subjective scores to accommodate for the quality rating compression at the extremes of the test range [10].

PSNR, SSIM, Proposed Metric (VPM) vs. MOS disperse points are showed in fig.4. Curve line in fig.4 is got by logistic fitting function. From fig.4, it can draw a conclusion that the proposed Metric (VPM) could assesst image quality with reliability and validity.
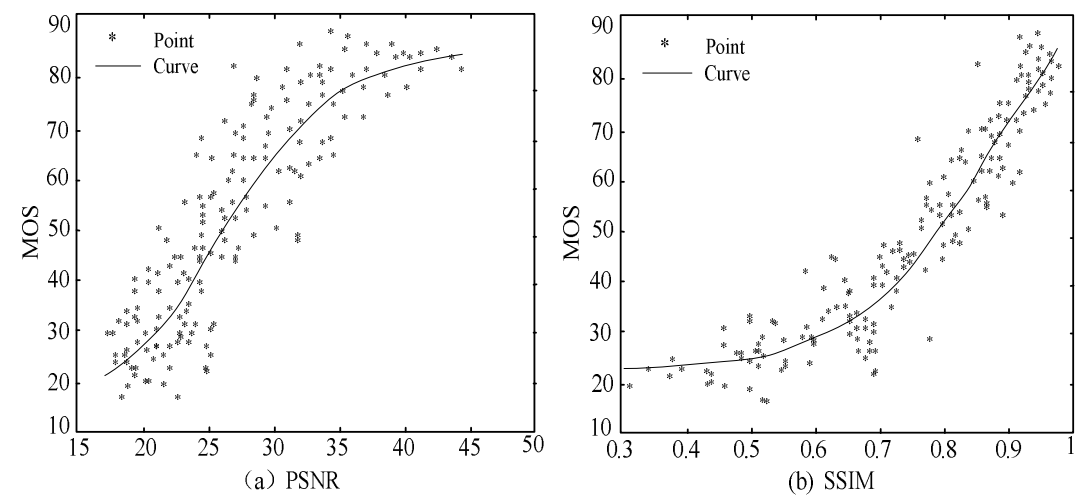

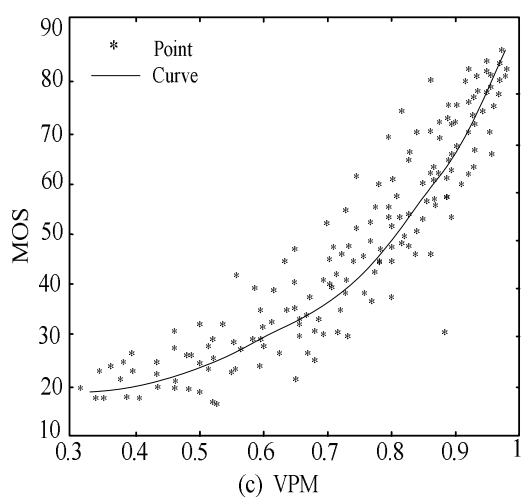

(c) VPM

Fig.4 PSNR, SSIM, Proposed Metric (VPM) vs. MOS Disperse Points

VQEG suggest that using three relativety fuctions to test objective/subjective scores . Variance weighted correlation, Spearman rank-order correlation and Outlier ratio. Table 1 gives evaluation of metrics peformance when appied to gaussian blur. Parameter value in the Table 1 prove that proposed metric (VPM) is excellent than PSNR metric and equate to SSIM metric. SSIM metric is full-reference image quality evaluation, while the proosed Metric (VPM) is no-reference image quality evaluation. 
Table 1. Evaluation of Metrics Peformance When Appied to Gaussian Blur

\begin{tabular}{lccc}
\hline Metric & Variance weighted correlation & Spearman correlation & Outlier ratio \\
\hline PSNR & 0.9042 & 0.8654 & 0.2325 \\
SSIM & 0.9578 & 0.9378 & 0.1048 \\
VPM & 0.9482 & 0.9323 & 0.1204 \\
\hline
\end{tabular}

\section{Conclusions}

In this paper, we have proposed a new no-reference objective sharpness metric algorithm based on visual perception. The algorithm is a combination of the spatial domain edge profile acutance and human visual characteristics. It gets the parameters of indicate the edge profile acutance by calculating the gradients in typical edge. The result indicate that the proposed image sharpness metric is used well for no-reference image sharpness metrics and achieves performance closer to the full-reference metric. This metric can be easily extended to measure the degree of sharpness in image captured from optical tracking and measure system.

\section{Acknowledgement}

This research is supported in part by Natural Science Foundation of China (No. 61007012), by general administration of quality subversion of china (No. 2008QK327) and by Education Department Foundation of Zhejiang provincial (No. Y200805868).

\section{References}

[1] Z. Wang, H.R. Sheikh, A.C. Bovik, "No-reference perceptual quality assessment of JPEG compressed images", Proceedings of the ICIP' 02, 477-480. (2002)

[2] J.E. Caviedes, F. Oberti, "No-reference quality metric for degraded and enhanced video", Proceedings of VCIP 2003, Lugana Switzerland, 6, 621-632. (2003)

[3] S. Suresh, R. Venkatesh Babu, H.J. Kim, "No-reference image quality assessment using modified extreme learning machine classifier ", Applied Soft Computing, 9, 541-552 (2009).

[4] M. Masry, S. Hemami, Y. Sermadevi, "A scalable wavelet based video distortion metric and applications", IEEE Trans. Circuits Systems Video Technol. 16 (2):260 - 273. (2006)

[5] Z.M. Parvez Sazzad, Y.Kawayoke, "No reference image quality assessment for JPEG2000 based on spatial features", Signal Processing: Image Communication 23, 257- 268 (2008).

[6] R. Venkatesh Babua, S. Sureshb, Andrew Perkis, "No-reference JPEG-image quality assessment using GAP-RBF", Signal Processing 87, 1493-1503(2007).

[7] Deepak S. Turaga, Yingwei Chen, Jorge Caviedes, "No reference PSNR estimation for compressed pictures", Signal Processing: Image Communication 19, 173-184(2004).

[8] R. Sheikh, Z. Wang, L. Cormack and A. C. Bovik, "LIVE Image Quality Assessment Database Release 2", http://live.ece.utexas.edu/research/quality.

[9] VQEG, Final Report from the Video Quality Experts Group on the Validation of Objective Models of Video Quality Assessment, Mar. 2000[Online]. Available: http:/www.vqeg.org/

[10] Rony Ferzli, Lina J. Karam. A No-Reference Objective Image Sharpness Metric Based on the Notion of Just Noticeable Blur (JNB), IEEE Translation on Image Processing, Vol. 18, No. 4, April 2009 